\title{
Identification of potential functional genes in papillary thyroid cancer by co-expression network analysis
}

\author{
ZENG-XIN AO ${ }^{1}$, YUAN-CHENG CHEN $^{1}$, JUN-MIN LU $^{1}$, JIE SHEN $^{1}$, LIN-PING PENG $^{1}$, XU LIN $^{1}$, \\ CHENG PENG $^{1}$, CHUN-PING ZENG ${ }^{1}$, XIA-FANG WANG ${ }^{1}$, ROU ZHOU ${ }^{1}$, ZHI CHEN $^{1}$, \\ HONG-MEI XIAO ${ }^{2}$ and HONG-WEN DENG ${ }^{1-3}$ \\ ${ }^{1}$ Department of Endocrinology and Metabolism, The Third Affiliated Hospital, Southern Medical University, Guangzhou, \\ Guangdong 510630; ${ }^{2}$ School of Basic Medical Sciences, Central South University, Changsha, Hunan 410000, P.R. China; \\ ${ }^{3}$ Department of Biostatistics and Bioinformatics, Center for Bioinformatics and Genomics, \\ Tulane University, New Orleans, LA 70112, USA
}

Received March 1, 2018; Accepted June 12, 2018

DOI: $10.3892 / \mathrm{ol} .2018 .9306$

\begin{abstract}
Interactions between multiple genes are involved in the development of complex diseases. However, there are few analyses of gene interactions associated with papillary thyroid cancer (PTC). Weighted gene co-expression network analysis (WGCNA) is a novel and powerful method that detects gene interactions according to their co-expression similarities. In the present study, WGCNA was performed in order to identify functional genes associated with PTC using R package. First, differential gene expression analysis was conducted in order to identify the differentially expressed genes (DEGs) between PTC and normal samples. Subsequently, co-expression networks of the DEGs were constructed for the two sample groups, respectively. The two networks were compared in order to identify a poorly preserved module. Concentrating on the significant module, validation analysis was performed to confirm the identified genes and combined functional enrichment analysis was conducted in order to identify more functional associations of these genes with PTC. As a result, 1062 DEGs were identified for network construction. A brown module containing 118 highly related genes was selected as it exhibited the lowest module preservation. After validation analysis, 61 genes in the module were confirmed to be associated with PTC. Following the enrichment analysis, two PTC-related pathways were identified: Wnt signal pathway and transcriptional misregulation in cancer. LRP4, KLK7, PRICKLE1, ETV4 and ETV5 were predicted to be candidate genes regulating the pathogenesis of PTC. These
\end{abstract}

Correspondence to: Professor Hong-Wen Deng, Department of Biostatistics and Bioinformatics, Center for Bioinformatics and Genomics, Tulane University, 1440 Canal Street, New Orleans, LA 70112, USA

E-mail: hdeng2@tulane.edu

Key words: papillary thyroid cancer, co-expression, gene interactions, LRP4, KLK7, PRICKLE1, ETV4, ETV5 results provide novel insights into the etiology of PTC and the identification of potential functional genes.

\section{Introduction}

Thyroid cancer is the most common type of endocrine malignancy. The incidence of thyroid cancer has increased markedly worldwide in the last decade (1). Papillary thyroid cancer (PTC) is the most common type of thyroid cancer, accounting for approximately $80 \%$ of all cases (2). Understanding of the genetic mechanisms underlying PTC has improved remarkably in recent years, which provides more support for molecular diagnosis of the disease $(3,4)$. However, predicting PTC as a result of the complicated molecular interactions participating in the pathogenesis of this type of cancer remains a clinical challenge (5). In addition, a number of patients with PTC demonstrate recurrence, invasion and distant metastasis due to resistance to surgical and radioiodine treatment. Novel molecular-targeted treatments hold potential for these cases (6). Therefore, investigation of the molecular mechanisms involved is expected to aid the identification of candidate biomarkers and improve molecular diagnosis and treatment of patients with PTC.

Previous studies of the genetic basis of PTC mainly focus on a single susceptibility gene, which does not take into account the interactions of multiple genes. However, complex traits are modified by the cumulative effect of interactive genes (7). Elucidation of the interactions between genes and molecules is essential for understanding the molecular basis underlying complex diseases. Correlation network analysis identifies the correlation patterns among genes according to their co-expression similarities by grouping highly correlated genes into one module (8). Another advantage of network-assisted analysis is that transcriptional data has a more direct impact on protein expression than genomic data, which provides more biological information regarding the interactive genes (9). The method applied in the present study is weighted gene co-expression network analysis (WGCNA) (8), which has been successfully used for detection of genetic determinants in many human diseases, such as osteoporosis (10), obesity (11), 
familial combined hyperlipidemia (12) and metaphyseal chondrodysplasia (13).

A gene harboring changeable interactions with other genes between diseased and normal conditions indicates that it may play a crucial role in pathology of the disease. Based on this hypothesis, Riquelme Medina and Lubovac-Pilav successfully employed WGCNA to identify genes and pathways involved in the development of type 1 diabetes (14). In the present study, WGCNA is employed to generate two networks derived from PTC and normal tissue control samples, respectively. The two networks were compared in order to identify the module with the lowest preservation, which contains genes harboring changeable interactions. To the best of our knowledge, the present study is the first to apply WGCNA innovatively in order to identify the genetic mechanisms of PTC.

\section{Materials and methods}

Gene expression data and processing. The gene expression dataset (accession number: GSE33630) was downloaded from the NCBI Gene Expression Omnibus database (http://www.nibi.nih.gov/geo/). Microarray data were obtained from RNA samples comprised of 49 PTC samples and 45 adjacent normal thyroid samples. The research was approved by Ehics Committees of the Institute of Endocrinology and Metabolism in Kiev, Imperial College in London and the Medical Radiological Research Centre in Obninsk. Details about the data can be found in the original publication (15). The platform used for profiling was the Affymetrix U-133 Plus v.2.0 array. The raw CEL data were processed and normalized using affy package in R software 3.3.0 (16). The probe IDs were converted into gene symbols using the annotation file for probes of the platform. The median expression levels of these probe IDs that correspond to the same gene were selected as the final expression value of a gene.

Differential gene expression analysis. In order to identify differentially expressed genes (DEGs) between the PTC and normal samples, empirical Bayes method was performed using limma package (17) in R. P-values were adjusted using the Benjamini and Hochberg method to control the false discovery rate caused by multiple testing. Genes were selected as DEGs for subsequent analysis according to the following criteria: $\log \mathrm{FC}$ (fold change) $>1$ and adjusted P-value $<0.05$. Fold change was calculated as the ratio of the differential expression.

Construction of weighted gene co-expression networks. Network construction was performed using the WGCNA $\mathrm{R}$ package $(8,18)$. First, cluster sampling was conducted for both the PTC and normal samples based on the expression levels of the DEGs to identify and remove potential outliers. The parameter beta of power function for network construction was then set as 8 according to the scale-free topology criterion as described in a previous study (8), making the established network satisfy approximate scale-free topology (linear regression model-fitting index R2 $>0.9$ ). WGCNA identifies modules with highly correlated genes; these genes usually have similar connectivity patterns with other genes, which can be defined as the topological overlap measurement (TOM). Genes were hierarchically clustered and visualized in a dendrogram according to dissimilarity TOM (1-TOM) (18). The branches of the tree representing highly correlated genes were grouped into one module marked by a particular color. Grey donates background genes that belonged to none of the modules. Different numbers of modules were established from the dendrogram in the PTC and normal samples, respectively.

Module preservation between PTC and normal networks. Module preservation analysis is capable of assessing whether a module defined in a reference dataset (PTC sample network in the present study) is also in the test dataset (normal sample network in the present study). Two composite preservation statistics, $Z_{\text {summary }}$ (Eq1) and medianRank (Eq2), were used to compare relative preservation among multiple modules, which considers both density and connectivity preservation (19). The former is based on the extent of gene interconnectivity, while the latter is based on the connectivity pattern of genes.

$$
\mathrm{Z}_{\text {summary }}=\left(\mathrm{Z}_{\text {density }}+\mathrm{Z}_{\text {connectivity }}\right) / 2(\mathrm{Eq1})
$$

medianRank $=($ medianRank.density + medianRank.connectivity)/2 (Eq2).

Studies have shown that $Z_{\text {summary }}$ thresholds distinguish module preservation as follows (19): $Z_{\text {summary }}<2$, no preservation; $Z_{\text {summary }}>10$, strong evidence of module preservation; and $2<Z_{\text {summary }}<10$, weak to moderate evidence of preservation. Modules with lower $Z_{\text {summary }}$ values are expected to be less preserved. MedianRank is a rank-based measurement that relies on a observed preservation statistic that shows no dependence on modulesize. A higher medianRank measurement indicates that the module exhibits less preservation. Modules of poor preservation are significant modules that perturbate important pathways and biological processes when comparing diseased and normal networks.

Intramodule gene interaction analysis. K.in, also known as intramodule connectivity, describes the connectivity of a gene with other genes in the same module (10). The k.in rank of a gene is the ranking of $k$.in among all of the genes in a particular module. K.in rank change refers to the difference of the k.in ranks between the PTC and normal sample networks. A gene with a large k.in rank change represents a large difference in its interactions with other genes in PTC and normal samples and indicates that it has an important role in the development of PTC.

Validation analysis using another gene expression dataset. In order to partially validate the identified genes in the significant module, a gene expression validation analysis was performed using another gene expression profile. The expression array for the validation was downloaded from the NCBI Gene Expression Omnibus database (accession number: GSE3678) and was derived from 7 PTC samples and 7 normal thyroid control samples. The point-biserial correlation coefficient was employed to estimate the relationship between PTC and gene expression levels. After 1000 bootstrap replications, the 

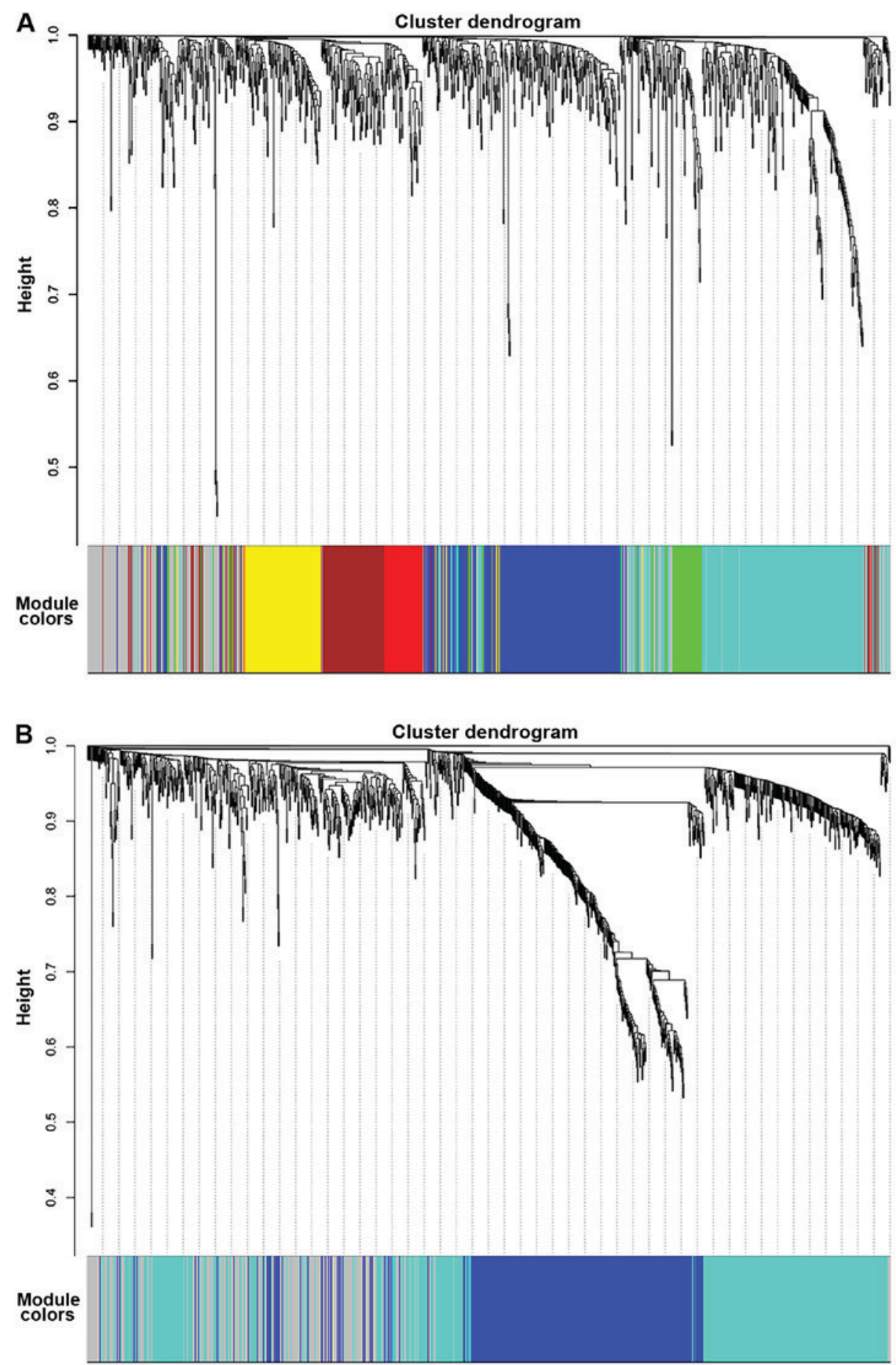

Figure 1. The cluster dendograms for (A) PTC sample network and (B) normal sample network. Each branch (vertical line) represents an individual gene. Each module is marked by a particular color which contains highly correlated genes. PTC, papillary thyroid cancer.

average of the correlation coefficients was calculated as the final point-biserial correlation coefficient of each gene.

Functional and pathway enrichment analyses of the significant module. Functional annotation of the genes in the significant module was accomplished using the online tool Database for Annotation Visualization and Integrated Discovery (DAVID) (http://david.abcc.ncifcrf.gov/) (20), depending on the GO term database and pathway database. Pathway enrichment of the module of interest was performed with another online tool, ConcensusPathDB (http://cpdb.molgen.mpg.de/) (21), based on the database of KEGG. This step evaluated the potential function of the genes related to thyroid carcinomas according to the known databases and known molecular pathways.

\section{Results}

Differential gene expression analysis. A total of 1062 genes were identified as DEGs for network construction based on the aforementioned criteria. The 1062 genes consisted of 653 up-regulated genes and 409 down-regulated genes in the PTC samples compared with the normal samples. 


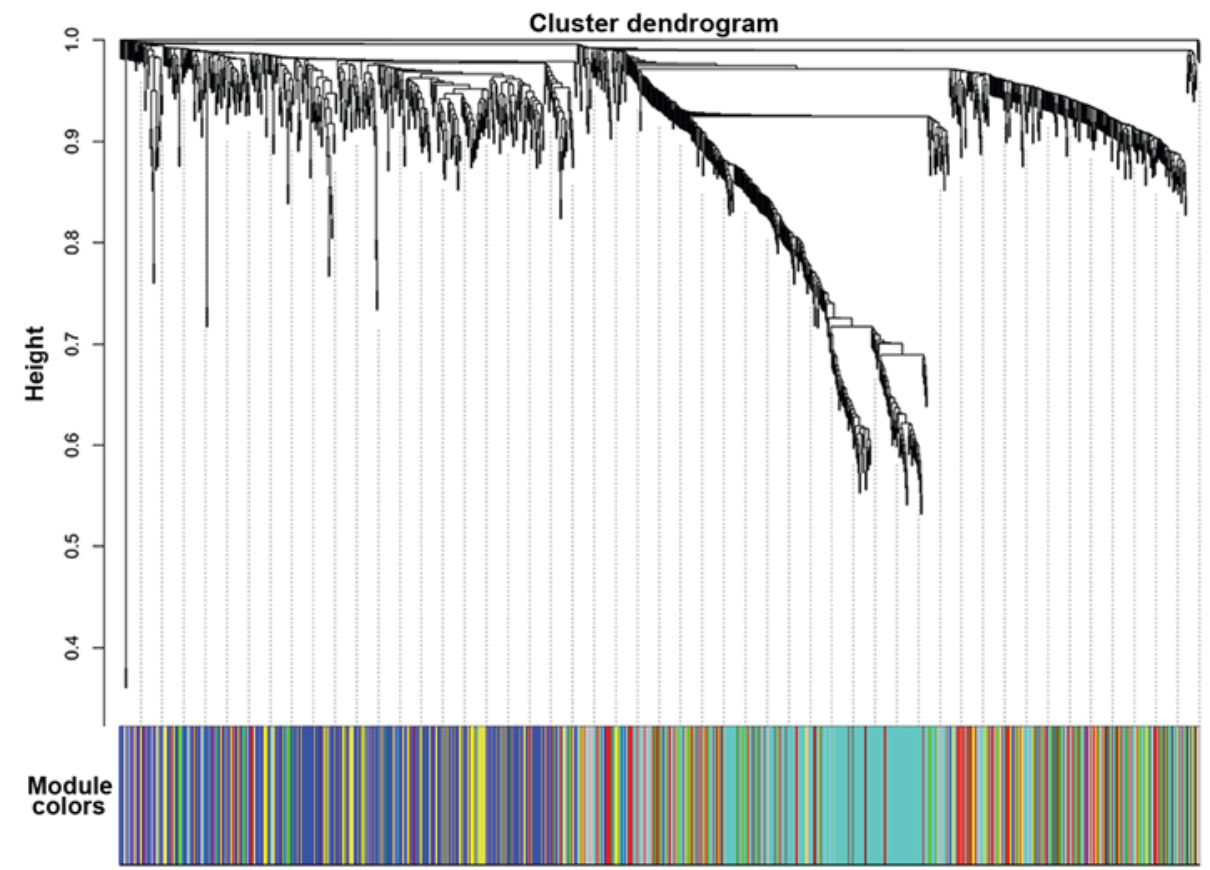

Figure 2. Visual change between PTC sample network and normal sample network. As a result, integrated modules in PTC sample network were divided into several parts in normal sample network. PTC, papillary thyroid cancer.
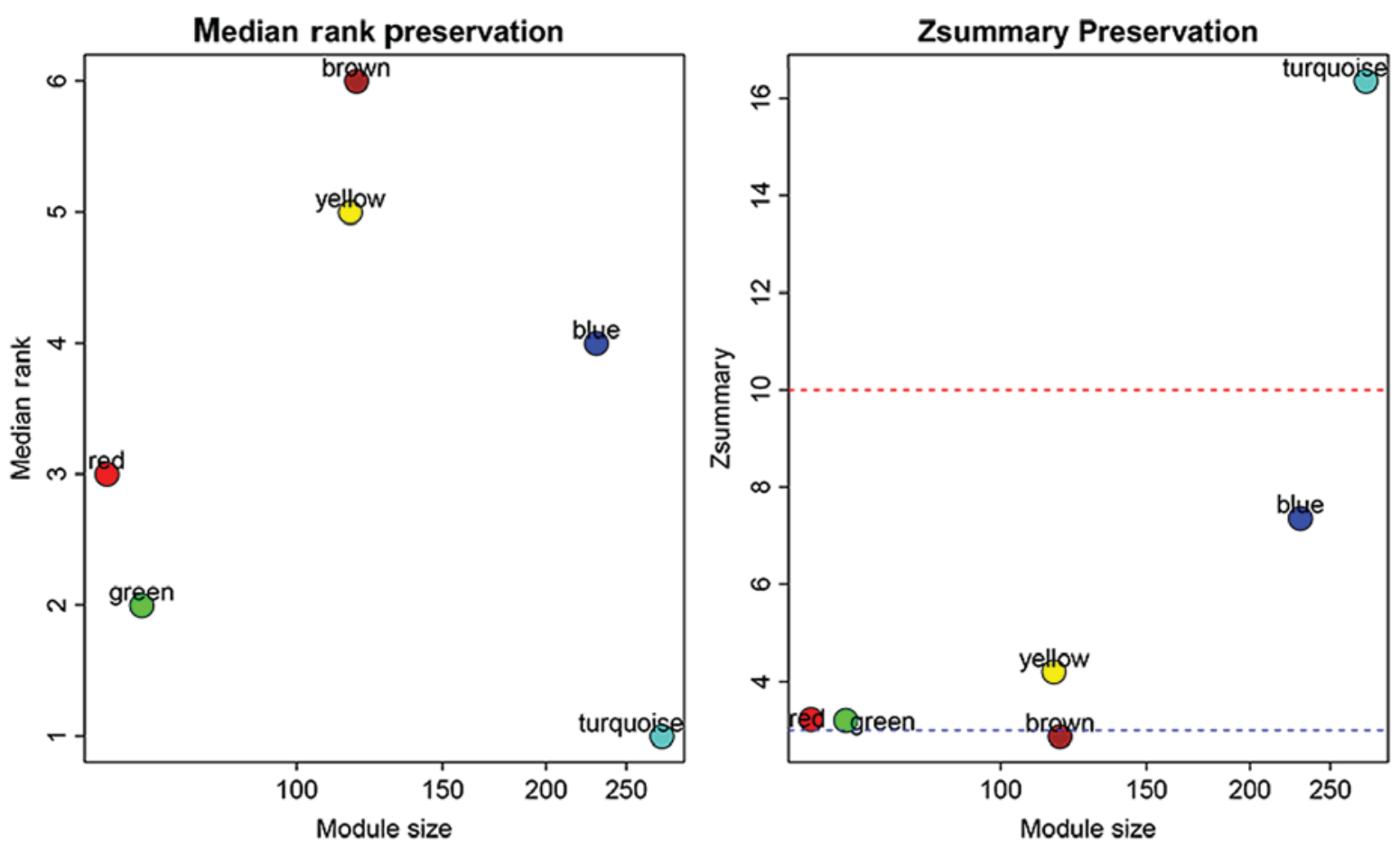

Figure 3. Preservation of modules in normal sample network compared with PTC sample network. The left panel shows the statistic medianRank vs. module size. The right panel shows the composite statistic Zsummary vs. module size. PTC, papillary thyroid cancer.

Network construction for the PTC and normal samples with WGCNA. WGCNA R package was applied in order to generate two different co-expression networks for the two sample groups with the 1062 DEGs. Hierarchically clustered dendrograms were generated and modules containing highly interconnected genes were detected. Seven modules, represented by blue, brown, green, grey, red, turquoise and yellow, were detected in the PTC samples, with module sizes of 230, 118, 65, 198, 59, 276 and
116 genes, respectively. Three modules were identified in the normal samples, represented by blue, grey and turquoise, which contained 358, 240 and 464 genes, respectively. The gene cluster dendrograms and detected modules are presented in Fig. 1.

Comparison of networks between the PTC and normal samples. In order to visualize the difference between the two networks, the same color of the module that each gene 
Table I. Validation results of the significant genes with top20 k.in rank change values.

\begin{tabular}{lccc}
\hline Gene symbol & $\mathrm{r}^{\mathrm{a}}$ & P-value & $\begin{array}{c}\text { k.in rank } \\
\text { change }^{\mathrm{b}}\end{array}$ \\
\hline LRP4 & 0.744359743 & $8.71 \times 10^{-05}$ & 108 \\
TMEM108 & 0.663751001 & 0.001446 & 94 \\
ETV4 & 0.801399494 & $5.13 \times 10^{-06}$ & 89 \\
CAPN3 & 0.751756977 & $6.33 \times 10^{-05}$ & 79 \\
KLK7 & 0.808517499 & $3.36 \times 10^{-06}$ & 76 \\
TNRC6C-AS1 & 0.864710246 & $5.44 \times 10^{-08}$ & 75 \\
FXYD5 & 0.676025828 & 0.001009 & 74 \\
KCNK5 & 0.673939248 & 0.001074 & 73 \\
ST3GAL5 & 0.761898241 & $4.00 \times 10^{-05}$ & 72 \\
KLK10 & 0.789168637 & $1.02 \times 10^{-05}$ & 71 \\
PRICKLE1 & 0.747557852 & $7.60 \times 10^{-05}$ & 69 \\
BCHE & -0.514997779 & 0.031851 & 68 \\
NELL2 & 0.589338467 & 0.008726 & 68 \\
IGFBP6 & 0.565886466 & 0.013735 & 61 \\
LOC102724312 & 0.718083931 & 0.000247 & 61 \\
LPL & 0.833774975 & $6.39 \times 10^{-07}$ & 61 \\
SLIT1 & 0.568912151 & 0.012987 & 61 \\
MYH10 & 0.785351749 & $1.25 \times 10^{-05}$ & 59 \\
LGALS1 & 0.500150827 & 0.039436 & 57 \\
LRRK2 & 0.843001019 & $3.24 \times 10^{-07}$ & 57 \\
& & & \\
\hline
\end{tabular}

${ }^{a}$ Point-biserial correlation coefficients; ${ }^{b}$ the difference of k.in rank between PTC and normal sample network. PTC, papillary thyroid cancer.

belonged to in the PTC sample network was applied to the corresponding gene in the normal sample network, without changing the hierarchically clustered dendrogram of the normal sample network. Visual change of the module structure is presented in Fig. 2. Integrated modules in the PTC sample network were found to be divided into several parts in the normal sample network.

To objectively estimate whether a module is preserved, $Z_{\text {summary }}$ and medianRank were employed in order to investigate the module preservation quantitatively. Visual results of the module preservation analysis including the statistics medianRank and $Z_{\text {summary }}$ are presented in Fig. 3. The brown module has the highest medianRank as well as the lowest $Z_{\text {summary }}$ among all the modules, indicating that it has the strongest preservation of all the modules. However, the $Z_{\text {summary }}$ value of the brown module is $<3$, which indicates no preservation according to the criterion of $Z_{\text {summary }}$. The brown module was selected for further analysis, when its medianRank and $Z_{\text {summary }}$ statistics were both taken into account.

Identification of genes associated with PTC in the brown module. The k.in rank change was calculated for each gene in the brown module in order to detect gene interaction changes when PTC occurs. After validation of the 118 genes in the brown module using point-biserial correlation coefficient, 61 genes were confirmed to be significantly relevant to
PTC with P-value $<0.05$. The validation results of the significant genes with the top $20 \mathrm{k}$.in rank change values are listed in Table I.

Functional annotation and enrichment analyses. The top10 significantly enriched terms in DAVID are shown in Table II, which includes extracellular region, integral component of plasma membrane, negative regulation of ERK1 and ERK2 cascade, extracellular space and cell-cell signaling.

Table III shows the results of pathway enrichment based on the KEGG database with the top5 enriched pathways. Several significantly enriched pathways are expected to be involved in PTC, such as transcriptional misregulations in cancer and Wnt signaling pathway $(22,23)$.

\section{Discussion}

Though studies of genomic events concerning the genetic mechanisms of PTC have achieved great progress in recent years $(3,4)$, few of them have noted interactions of correlated genes thus far. Correlation network-based analysis is a novel method for investigation of biologically interactive genes associated with complex disease. Network analysis groups interactive genes into one module in which genes are enriched for specific biological pathways. Moreover, expression data used for gene grouping are from tissues and cells involved in the disease, so the interconnections between genes are only revealed in the specific disease conditions.

Previous studies using expression data mainly focus on the DEGs in order to identify potential genes involved in PTC (24-27). In the present study, in addition to performing differential gene expression analysis, co-expression networks were established for the PTC and normal samples, respectively, using the 1062 DEGs identified. Comparison of gene-gene interaction between normal and disease networks is useful for identifying dysregulated pathways in the process of disease. This method enhances the ability to detect functional genes with regards to changing crosstalk with other genes rather than merely changing expression levels. Module preservation analysis was applied in the present study and a poorly preserved module comprised of 118 interactive genes was identified.

Analyzing the genes in the brown module, the k.in rank change for each gene was calculated in order to detect gene interaction changes when PTC occurs. Subsequently, 61 genes were validated to be significantly relevant to PTC in the validation analysis. LRP4 is a promising candidate gene for PTC as it ranks first based on the k.in rank changes among the 118 genes in the brown module and also has a high correlation coefficient $(\mathrm{r}=0.7444)$ with PTC. In addition, it has been demonstrated to be associated with PTC in previous studies.LRP4 is reported to be overexpressed in PTC samples (28) and is included in a molecular classifier that is able to discriminate thyroids with PTC accurately from normal thyroids (29). KLK7 is also a potential oncogene for PTC. KLK7 has the fifth largest k.in rank change, as shown in Table $\mathrm{I}$, as well as a significant correlation coefficient $(\mathrm{r}=0.8085)$ with PTC in the validation analysis. KLK7 has highly increased levels of expression in PTC compared with those in normal tissue (30). Furthermore, a previous study suggested that KLK7 promotes tumorgenesis and progress in PTC in vitro and in vivo experiments (31). 
Table II. Module functional enrichment in DAVID.

\begin{tabular}{|c|c|c|c|c|}
\hline Category & GO ID & GO term & No. of genes & P-value \\
\hline $\mathrm{CC}$ & GO:0005576 & Extracellular region & 21 & $5.4 \times 10^{-04}$ \\
\hline $\mathrm{CC}$ & GO:0005887 & Integral component of plasma membrane & 19 & $8.1 \times 10^{-04}$ \\
\hline $\mathrm{BP}$ & GO:0070373 & Negative regulation of ERK1 and ERK2 cascade & 4 & $4.1 \times 10^{-03}$ \\
\hline $\mathrm{BP}$ & GO:0051965 & Positive regulation of synapse assembly & 4 & $4.9 \times 10^{-03}$ \\
\hline $\mathrm{CC}$ & GO:0005615 & Extracellular space & 16 & $7.9 \times 10^{-03}$ \\
\hline $\mathrm{BP}$ & GO:0060976 & Coronary vasculature development & 3 & $8.4 \times 10^{-03}$ \\
\hline $\mathrm{BP}$ & GO:0016042 & Lipid catabolic process & 4 & $1.2 \times 10^{-02}$ \\
\hline $\mathrm{BP}$ & GO:0007411 & Axon guidance & 5 & $1.2 \times 10^{-02}$ \\
\hline BP & GO:0007267 & Cell-cell signaling & 6 & $1.3 \times 10^{-02}$ \\
\hline $\mathrm{MF}$ & GO:0005198 & Structural molecule activity & 6 & $1.5 \times 10^{-02}$ \\
\hline
\end{tabular}

$\mathrm{GO}$, gene ontology; CC, cellular components; $\mathrm{BP}$, biological processes; MF, molecular functions.

Table III. Pathway enrichment in ConsensusPathDB.

\begin{tabular}{lcll}
\hline Pathway name & No. of genes & & Genes \\
\hline Taste transduction & 3 & KCNK5, CHRM3, GABBR2 & 0.0104 \\
Transcriptional misregulation in cancer & 4 & ETV4, ETV5, PLAU, DUSP6 & 0.0164 \\
Regulation of actin cytoskeleton & 4 & MYH10, RRAS, CHRM3, CYFIP2 & 0.0288 \\
Wnt signal pathway & 3 & CCND1, PRICKLE1, WIF1 & 0.0424 \\
Rennin secretion & 2 & ADORA1, KCNJ2 & 0.0489
\end{tabular}

To further investigate the functional relevance of the identified genes in PTC, functional annotation and enrichment analyses were performed and two significant pathways which may play a role in pathogenesis of PTC were identified. One of the significant pathways is the Wnt signal pathway, which has been implicated in the genesis and development of PTC in previous studies $(22,23)$. CCND1 and PRICKLE1, which are enriched in this pathway in the enrichment analysis, have both been shown to be significantly relevant in PTC with high correlation coefficient values $(r=0.8263$ and $r=0.7476$, respectively) in the validation analysis. CCND1 has been reported to be a vital gene in PTC. Studies have shown that CCND1 expression is significantly associated with PTC growth and lymph node metastases through aberrant activation of the Wnt/beta-catenin pathway $(32,33)$. PRICKLE1 is potential gene associated with PTC. PRICKLE1 encodes prickle planar cell polarity (PCP) protein land is a core signaling molecule in the Wnt/PCP signaling pathway $(34,35)$. Previous studies indicate that the Wnt/PCP signaling pathway plays critical roles in the proliferation and migration of tumor cells $(36,37)$. The other pathway identified to be involved in PTC is transcriptional misregulation in cancer. ETV4, ETV5 and DUSP6, which are enriched in this pathway, were all confirmed to be relevant to PTC in the validation analysis.DUSP6 has been reported to play an essential role in the initiation and progression of PTC by many functional experiments (38-40). ETV4 and ETV5 are both oncogenic E26 transformation-specific family transcription factors (41) and are implicated in many other types of cancer, such as colorectal (42), prostatic (43), endometrial (44) and ovarian (45) cancer. The results of the present study provide novel direction for better understanding the molecular mechanisms involved in PTC.

The present study has several limitations. Firstly, not all of the genes associated with PTC that have been reported in previous genetic studies were identified as the present study is only a supplement to the current investigations of the genetic mechanism of PTC. However, previous studies often focus on the effect of a single gene on PTC, while the present study highlights the impact of gene-gene interactions on the tumor pathological process, which provides a novel insight for detection of potential functional genes. Secondly, the exact effects of the identified genes require corroboration with biological experiments. However, validation and enrichment analyses were used to confirm the identified genes in the present study. Certain genes, such as CCND1 and DUSP6, were confirmed that have been shown to be involved in the pathology of PTC by previous functional and experimental studies.

In conclusion, the present study applied correlated network analysis in order to concentrate on gene interactions. Identified genes were validated using another set of expression data and then functional enrichment analysis was used to identify more biological associations. A number of novel genes, such as LRP4, KLK7, PRICKLE1, ETV4 and ETV5, and pathways (Wnt signal pathway and transcriptional misregulation in cancer) were identified that may exert important functions in PTC. These results contribute to the 
understanding of the genetic basis of PTC and provide novel insights into the identification of potential functional genes in PTC.

\section{Acknowledgements}

Not applicable.

\section{Funding}

The present study partially supported by grants from The National Institutes of Health (grant nos. R01AR069055, U19 AG055373, R01 MH104680, R01AR059781 and P20 GM109036); The Edward G. Schlieder Endowment fund from Tulane University; The National Natural Science Foundation of China (grant no. 81302228); The Foundation for Pearl River Nova program of Guangzhou (grant no. 2014J2200034); and The Technological Innovation Project of Foshan City (grant no. 2017AG100102).

\section{Availability of data and materials}

The datasets used during the current study are available in the NCBI Gene Expression Omnibus database, with the accession numbers are GSE33630 and GSE3678.

\section{Authors' contributions}

HWD, JS, ZC and HMX conceived and designed the study. ZXA, YCC, JML, LPP and XL analyzed the data. ZXA, HWD, YCC, ZC and HMX wrote the manuscript. CP, CPZ, XFW and $\mathrm{RZ}$ collected the data and preprocessed the raw data.

\section{Ethics approval and consent to participate}

The research was approved by the Ethics Committees of the Institute of Endocrinology and Metabolism in Kiev, Imperial College in London and the Medical Radiological Research Centre in Obninsk.

\section{Patient consent for publication}

Not applicable.

\section{Competing interests}

The authors declare that they have no competing interests.

\section{References}

1. Kitahara CM and Sosa JA: The changing incidence of thyroid cancer. Nat Rev Endocrinol 12: 646-653, 2016.

2. Schneider DF and Chen H: New developments in the diagnosis and treatment of thyroid cancer. CA Cancer J Clin 63: 374-394, 2013.

3. Cancer Genome Atlas Research Network: Integrated genomic characterization of papillary thyroid carcinoma. Cell 159: 676-690, 2014.

4. Ye L, Zhou X, Huang F, Wang W, Qi Y, Xu H, Yang S, Shen L, Fei X, Xie J, et al: The genetic landscape of benign thyroid nodules revealed by whole exome and transcriptome sequencing. Nat Commun 8: 15533, 2017.

5. Hsiao SJ and Nikiforov YE: Molecular approaches to thyroid cancer diagnosis. Endocr Relat Cancer 21: T301-T313, 2014.
6. Xing M, Haugen BR and Schlumberger M: Progress in molecular-based management of differentiated thyroid cancer. Lancet 381: 1058-1069, 2013.

7. Eichler EE, Flint J, Gibson G, Kong A, Leal SM, Moore JH and Nadeau JH: Missing heritability and strategies for finding the underlying causes of complex disease. Nat Rev Genet 11: 446-450, 2010.

8. Langfelder P and Horvath S: WGCNA: An R package for weighted correlation network analysis. BMC Bioinformatics 9: $559,2008$.

9. Liu W and Ye H: Co-expression network analysis identifies transcriptional modules in the mouse liver. Mol Genet Genomics 289: 847-853, 2014.

10. Chen YC, Guo YF, He H, Lin X, Wang XF, Zhou R, Li WT, Pan DY, Shen J and Deng HW: Integrative analysis of genomics and transcriptome data to identify potential functional genes of BMDs in females. J Bone Miner Res 31: 1041-1049, 2016.

11. Kogelman LJ, Cirera S, Zhernakova DV, Fredholm M, Franke L and Kadarmideen HN: Identification of co-expression gene networks, regulatory genes and pathways for obesity based on adipose tissue RNA sequencing in a porcine model. BMC Med Genomics 7: 57, 2014.

12. Plaisier CL, Horvath S, Huertas-Vazquez A, Cruz-Bautista I, Herrera MF, Tusie-Luna T, Aguilar-Salinas C and Pajukanta P: A systems genetics approach implicates USF1, FADS3, and other causal candidate genes for familial combined hyperlipidemia. PLoS Genet 5: e1000642, 2009.

13. Wang B, He L, Miao W, Wu G, Jiang H, Wu Y, Qu J and Li M: Identification of key genes associated with Schmid-type metaphyseal chondrodysplasia based on microarray data. Int J Mol Med 39: 1428-1436, 2017.

14. Riquelme Medina I and Lubovac-Pilav Z: Gene Co-expression network analysis for identifying modules and functionally enriched pathways in type 1 diabetes. PLoS One 11: e0156006, 2016.

15. Tomás G, Tarabichi M,Gacquer D, Hébrant A,Dom G, Dumont JE, Keutgen X, Fahey TJ III, Maenhaut C and Detours V: A general method to derive robust organ-specific gene expression-based differentiation indices: Application to thyroid cancer diagnostic. Oncogene 31: 4490-4498, 2012.

16. Gautier L, Cope L, Bolstad BM and Irizarry RA: Affy-analysis of Affymetrix GeneChip data at the probe level. Bioinformatics 20: 307-315, 2004.

17. Smyth GK: Linear models and empirical bayes methods for assessing differential expression in microarray experiments. Stat Appl Genet Mol Biol 3: Article3, 2004.

18. Zhang B and Horvath S: A general framework for weighted gene co-expression network analysis. Stat Appl Genet Mol Biol 4: Article17, 2005.

19. Langfelder P, Luo R, Oldham MC and Horvath S: Is my network module preserved and reproducible? PLoS Comput Biol 7: e1001057, 2011

20. Huang DW, Sherman BT, Tan Q, Collins JR, Alvord WG, Roayaei J, Stephens R, Baseler MW, Lane HC and Lempicki RA: The DAVID gene functional classification tool: A novel biological module-centric algorithm to functionally analyze large gene lists. Genome Biol 8: R183, 2007.

21. Herwig R, Hardt C, Lienhard M and Kamburov A: Analyzing and interpreting genome data at the network level with ConsensusPathDB. Nat Protoc 11: 1889-1907, 2016.

22. Gilbert-Sirieix M, Makoukji J, Kimura S, Talbot M, Caillou B, Massaad C and Massaad-Massade L: Wnt $/ \beta$-catenin signaling pathway is a direct enhancer of thyroid transcription factor-1 in human papillary thyroid carcinoma cells. PLoS One 6: e22280, 2011.

23. Ishigaki $K$, Namba $H$, Nakashima $M$, Nakayama T, Mitsutake $N$, Hayashi T, Maeda S, Ichinose M, Kanematsu T and Yamashita S: Aberrant localization of beta-catenin correlates with overexpression of its target gene in human papillary thyroid cancer.JClinEndocrinol Metab 87: 3433-3440, 2002.

24. Fluge $\varnothing$, Bruland O, Akslen LA, Lillehaug JR and Varhaug JE: Gene expression in poorly differentiated papillary thyroid carcinomas. Thyroid 16: 161-175, 2006.

25. Qiu J, Zhang W, Xia Q, Liu F, Li L, Zhao S, Gao X, Zang C, Ge R and Sun Y: RNA sequencing identifies crucial genes in papillary thyroid carcinoma (PTC) progression. Exp Mol Pathol 100: 151-159, 2016.

26. Qu T, Li YP, Li XH and Chen Y: Identification of potential biomarkers and drugs for papillary thyroid cancer based on gene expression profile analysis. Mol Med Rep 14: 5041-5048, 2016. 
27. Zhu X, Yao J and Tian W: Microarray technology to investigate genes associated with papillary thyroid carcinoma. Mol Med Rep 11: 3729-3733, 2015.

28. Hucz J, Kowalska M, Jarzab M and Wiench M: Gene expression of metalloproteinase 11, claudin 1 and selected adhesion related genes in papillary thyroid cancer. Endokrynol Pol 57 (Suppl A): 18-25, 2006 (In Polish).

29. Jarzab B, Wiench M, Fujarewicz K, Simek K, Jarzab M, Oczko-Wojciechowska M, Wloch J, Czarniecka A, Chmielik E, Lange D, et al: Gene expression profile of papillary thyroid cancer: Sources of variability and diagnostic implications. Cancer Res 65: 1587-1597, 2005.

30. Kim HS, Kim DH, Kim JY, Jeoung NH, Lee IK, Bong JG and Jung ED: Microarray analysis of papillary thyroid cancers in Korean. Korean J Intern Med 25: 399-407, 2010

31. Zhang H, Cai Y, Zheng L, Zhang Z, Lin X and Jiang N: Long noncoding RNA NEAT1 regulate papillary thyroid cancer progression by modulating miR-129-5p/KLK7 expression. J Cell Physiol 233: 6638-6648, 2018.

32. Lamba Saini M, Weynand B, Rahier J, Mourad M, Hamoir M and Marbaix E: Cyclin D1 in well differentiated thyroid tumour of uncertain malignant potential. Diagn Pathol 10: 32, 2015

33. Lantsov D, Meirmanov S, Nakashima M, Kondo H, Saenko V, Naruke Y, Namba H, Ito M, Abrosimov A, Lushnikov E, et al: Cyclin D1 overexpression in thyroid papillary microcarcinoma: Its association with tumour size and aberrant beta-catenin expression. Histopathology 47: 248-256, 2005

34. Guo D, Yuan Z, Ru J, Gu X, Zhang W, Mao F, Ouyang H, Wu K, Liu Y and Liu C: A spatiotemporal requirement for prickle 1-mediated PCP signaling in eyelid morphogenesis and homeostasis. Invest Ophthalmol Vis Sci 59: 952-966, 2018.

35. Dyberg C, Papachristou P, Haug BH, Lagercrantz H, Kogner P Ringstedt T, Wickström $M$ and Johnsen JI: Planar cell polarity gene expression correlates with tumor cell viability and prognostic outcome in neuroblastoma. BMC Cancer 16: 259, 2016.

36. VanderVorst K, Hatakeyama J, Berg A, Lee H and Carraway KL III: Cellular and molecular mechanisms underlying planar cell polarity pathway contributions to cancer malignancy. Semin Cell Dev Biol: Nov 4, 2017 (Epub ahead of print).

37. Katoh M: WNT/PCP signaling pathway and human cancer (review). Oncol Rep 14: 1583-1588, 2005
38. Lee JU, Huang S, Lee MH, Lee SE, Ryu MJ, Kim SJ, Kim YK, Kim SY, Joung KH, Kim JM, et al: Dual specificity phosphatase 6 as a predictor of invasiveness in papillary thyroid cancer. Eur J Endocrinol 167: 93-101, 2012.

39. Degl'Innocenti D, Romeo P, Tarantino E, Sensi M, Cassinelli G, Catalano V, Lanzi C, Perrone F, Pilotti S, Seregni E, et al: DUSP6/MKP3 is overexpressed in papillary and poorly differentiated thyroid carcinoma and contributes to neoplastic properties of thyroid cancer cells. Endocr Relat Cancer 20: 23-37, 2013.

40. Gu Y, Li D, Luo Q, Wei C, Song H, Hua K, Song J, Luo Y, Li X and Fang L: MicroRNA-145 inhibits human papillary cancer TPC1 cell proliferation by targeting DUSP6. Int J Clin Exp Med 8: 8590-8598, 2015.

41. Kedage V, Selvaraj N, Nicholas TR, Budka JA, Plotnik JP, Jerde TJ and Hollenhorst PC: An interaction with ewing's sarcoma breakpoint protein EWS defines a specific oncogenic mechanism of ETS factors rearranged in prostate cancer. Cell Rep 17: 1289-1301, 2016.

42. Xiao J, Yang S, Shen P, Wang Y, Sun H, Ji F and Zhou D: Phosphorylation of ETV4 at Ser73 by ERK kinase could block ETV4 ubiquitination degradation in colorectal cancer. Biochem Biophys Res Commun 486: 1062-1068, 2017.

43. Qi M, Liu Z, Shen C, Wang L, Zeng J, Wang C, Li C, Fu W, Sun Y and Han B: Overexpression of ETV4 is associated with poor prognosis in prostate cancer: Involvement of uPA/uPAR and MMPs. Tumour Biol 36: 3565-3572, 2015.

44. Pedrola N, Devis L, Llauradó M, Campoy I, Martinez-Garcia E, Garcia M, Muinelo-Romay L, Alonso-Alconada L, Abal M, Alameda F, et al: Nidogen 1 and Nuclear Protein 1: Novel targets of ETV5 transcription factor involved in endometrial cancer invasion. Clin Exp Metastasis 32: 467-478, 2015.

45. Llauradó M, Majem B, Castellví J, Cabrera S, Gil-Moreno A, Reventós J and Ruiz A: Analysis of gene expression regulated by the ETV5 transcription factor in OV90 ovarian cancer cells identifies FOXM1 overexpression in ovarian cancer. Mol Cancer Res 10: 914-924, 2012.

This work is licensed under a Creative Commons

Attribution-NonCommercial-NoDerivatives 4.0 International (CC BY-NC-ND 4.0) License. 